\title{
Lévy Processes and Quasi-Shuffle Algebras
}

\author{
Charles Curry $^{\mathrm{a}}$, Kurusch Ebrahimi-Fard ${ }^{\mathrm{b}}$, Simon J.A. Malham ${ }^{\mathrm{a}}$ and Anke Wiese ${ }^{\mathrm{a} *}$ \\ ${ }^{a}$ Maxwell Institute for Mathematical Sciences and School of Mathematical and Computer \\ Sciences, Heriot-Watt University, Edinburgh EH14 4AS, UK; ${ }^{\mathrm{b}}$ Instituto de Ciencias \\ Matemáticas, Consejo Superior de Investigaciones Científicas, C/ Nicolás Cabrera, no. \\ 13-15, 28049 Madrid, Spain
}

(7 October 2013)

Accepted for publication in Stochastics: An International Journal of Probability and Stochastic

Processes

\begin{abstract}
We investigate the algebra of repeated integrals of semimartingales. We prove that a minimal family of semimartingales generates a quasi-shuffle algebra. In essence, to fulfill the minimality criterion, first, the family must be a minimal generator of the algebra of repeated integrals generated by its elements and by quadratic covariation processes recursively constructed from the elements of the family. Second, recursively constructed quadratic covariation processes may lie in the linear span of previously constructed ones and of the family, but may not lie in the linear span of repeated integrals of these. We prove that a finite family of independent Lévy processes that have finite moments generates a minimal family. Key to the proof are the Teugels martingales and a strong orthogonalization of them. We conclude that a finite family of independent Lévy processes form a quasi-shuffle algebra. We discuss important potential applications to constructing efficient numerical methods for the strong approximation of stochastic differential equations driven by Lévy processes.
\end{abstract}

Keywords: semimartingales, Lévy processes, quasi-shuffle algebra, Teugels martingales

AMS Subject Classification: 60H30, 60G44

\section{Introduction}

The set of all multiple Stratonovich integrals constructed from independent Wiener processes generates a shuffle algebra (see Gaines 1994). This is because the usual integration by parts formula holds for such multiple integrals. The set of all multiple Itô integrals on the other hand generates a quasi-shuffle algebra. In this case the non-zero quadratic variation of the underlying Wiener processes is revealed by the Itô integration by parts formula. As might be expected, the two algebras are isomorphic (by direct application of the results by Hoffman 2000). More generally, Li \& Liu 1997 studied algebraic bases for independent Wiener and Poisson processes and the set of multiple Itô integrals constructed from them.

Knowledge about the algebraic structure of stochastic systems has been proved to be very useful in a range of applications. Recent applications include chaotic representations of martingales (Jamshidian 2011), the generalization and study of the concept of a Fliess operator to input-output maps driven by Itô processes (Duffaut Espinosa, Gray \& González 2012), and the design and analysis of efficient

* Corresponding author. Email: A.Wiese@hw.ac.uk

Acknowledgements. KEF, SJAM and AW would like to thank the Edinburgh Mathematical Society for support for a visit by KEF to Heriot-Watt in July 2012. 
stochastic simulation methods for stochastic differential equations driven by Wiener processes (Malham \& Wiese 2009 and Ebrahimi-Fard et al. 2012), among others.

It is natural to now ask the question of whether a family of Wiener-Poisson processes or more generally of Lévy processes generates a quasi-shuffle algebra. Indeed what about a family of semimartingales? In this paper we prove the following new main results, that a collection of:

(1) Semimartingales that generate a minimal family (see Section 21) form a quasi-shuffle algebra;

(2) Lévy processes that have finite moments generate a minimal family.

A natural consequence is that the Hoffman exponential map (see Hoffman 2000 and Hoffman \& Ihara 2012) establishes an isomorphism between the quasi-shuffle algebra of Lévy processes and a shuffle algebra. From a practical strong simulation perspective knowledge of the quasi-shuffle structure is highly desirable. This is because in principle, we can utilize the convolution shuffle algebra analysis of Ebrahimi-Fard et al. (2012) to establish efficient strong integrators for stochastic differential equations driven by Lévy processes. Lévy processes have become increasingly popular in recent years and can now be regarded as one of the key ingredients for many models in finance and economics and in insurance; their efficient simulation has thus become an important aspect of these applications.

Let us outline the key points more explicitly. Suppose we are given a finite family of semimartingales. Without loss of generality we assume they are all zero at time $t=0$. The real product of two semimartingales $X$ and $Y$ is given by $X Y=\int X_{-} \mathrm{d} Y+\int Y_{-} \mathrm{d} X+[X, Y]$. Here $[X, Y]$ is the quadratic covariation of $X$ and $Y$ and represents the Itô correction to the classical integration by parts formula; it is itself a semimartingale, and the space of semimartingales with multiplication forms an algebra. The formula above is reminiscent of a quasi-shuffle of $X$ and $Y$, see Section 4, and see Hoffman 2000, Ebrahimi-Fard \& Guo 2006, Novelli, Patras \& Thibon 2011, and Hoffman \& Ihara 2012 for more details on this product. Indeed, for a minimal family of semimartingales, we can assign a letter to each element in the family, and inductively, new letters to those nested quadratic covariation processes that are new and not linear combinations of those hitherto constructed. In so doing, we define an alphabet $\mathbb{A}$, and we then establish an isomorphism from the quasi-shuffle algebra $\mathbb{R}\langle\mathbb{A}\rangle$ of noncommutative polynomials and formal power series generated by words formed from $\mathbb{A}$ to the algebra of repeated integrals generated by the given semimartingales.

Our second main result is that a family of independent Lévy processes with finite moments generates a minimal family. Key to establishing this result are Teugels martingales and a strong orthogonalization of them. Here we relied particularly on work by Nualart \& Schoutens (2000), Davis (2005), and Jamshidian (2005). We conclude a family of independent Lévy processes generates a quasi-shuffle algebra.

Our paper is structured as follows. We introduce our notion of minimal families of semimartingales in Section 2. In Section 3 we prove that a family of independent Lévy processes generate a minimal family. We characterize those Lévy processes for which the alphabet $\mathbb{A}$ is finite. With the concrete general example of independent Lévy processes in hand, we then establish in Section 4 the isomorphism from the quasi-shuffle algebra to the algebra generated by a minimal family of semimartingales. We then apply this result to a family of independent Lévy processes and derive further algebraic properties of the algebra generated by them. Finally in Section 5 we conclude and discuss important applications of our results. 


\section{Minimal families of semimartingales}

Underlying our analysis is a complete filtered probability space $\left(\Omega, \mathcal{F},\left(\mathcal{F}_{t}\right)_{t>0}, P\right)$ satisfying what is known as the usual conditions of completeness and rightcontinuity, see Protter (1992; p. 3). Without loss of generality, we assume that $\mathcal{F}_{0}$ is generated by the $P$-null sets. Due to the usual conditions every martingale has a modification that has paths that are right-continuous with left limits (see Protter p. 5, Corollary 1). We assume henceforth all martingales have this property.

A process $X$ is a semimartingale, if $X$ has a decomposition $X_{t}=X_{0}+M_{t}+A_{t}$ for $t \geq 0$, where $M_{0}=A_{0}=0$, and where $M$ is a local martingale and $A$ is an adapted process that is right-continuous with left limits and has finite variation on each finite interval $[0, t]$. Recall that a process $A$ is predictable, if it is measurable with respect to the $\sigma$-algebra on $\mathbb{R}^{+} \times \Omega$ generated by the left-continuous processes. A semimartingale $X$ that admits a decomposition with a predictable finite variation process $A$ is a special semimartingale. Such a decomposition is unique, see Jacod \& Shiryaev (2002, Definition I.3.1 and I.4.21).

The space of semimartingales with multiplication forms an algebra (Protter p. 60, Corollary 3). The quadratic covariation or square bracket process $[X, Y]$ between two semimartingales $X$ and $Y$ is defined via their product as follows

$$
X Y=X_{0} Y_{0}+\int X_{-} \mathrm{d} Y+\int Y_{-} \mathrm{d} X+[X, Y]
$$

see Protter (1992; p. 58). The quadratic covariation of a process $X$ with itself is known as its quadratic variation (we refer to the monographs by Protter (1992) and Jacod \& Shiryaev (2002) for details). The following property, which is essential for the definition of the quasi-shuffle product, follows from I.4.49 Proposition and I.4.52 Theorem in Jacod \& Shiryaev (2002).

Remark 1: Let $X, Y$ and $Z$ be semimartingales, then: (a) $[X, 0]=0$; (b) $[X, Y]=[Y, X]$; (c) $[X,[Y, Z]]=\sum_{s} \Delta X_{s} \Delta Y_{s} \Delta Z_{s}=[[X, Y], Z]$. Here $\Delta X_{s}:=$ $X_{s}-\lim _{u \nearrow_{s}} X_{u}$ denotes the jump of a semimartingale $X$ at time $s$.

Hence, the quadratic covariation defines a commutative, associative product on the space of semimartingales.

Let $\mathcal{X}=\left\{X^{1}, \ldots, X^{d}\right\}$ be a finite family of semimartingales. By considering $X^{i}-X_{0}^{i}$, we may and will assume $X_{0}^{i}=0$ for all $i=1, \ldots, d$. Let $\mathcal{A}$ denote the algebra of repeated integrals generated by $\mathcal{X}$. Important elements of the algebra $\mathcal{A}$ are the multiple bracket processes (see Jamshidian 2005).

Definition 2.1: (Power Bracket) For a semimartingale $X$, we denote $[X]^{(1)}=$ $X$, and we define the power bracket for $n \geq 2$ by $[X]^{(n)}=\left[X,[X]^{(n-1)}\right]$.

Note for $n \geq 3$, the $n$-bracket is given by $[X]^{(n)}=\sum(\Delta X)^{n}$. This is also known as the power jump process (see Nualart \& Schoutens 2000). Key to relating the semimartingale product to a quasi-shuffle product is the following property.

Definition 2.2: (Minimal family) A family $\mathcal{X}$ of semimartingales is minimal if it satisfies the following two conditions:

(A) (Minimal Generator) It is the minimal generator of the algebra $\mathcal{A}$ of repeated integrals generated by $\mathcal{X}$ and by successively constructed nested covariation processes from $\mathcal{X}$.

(B) (Consistency) Successively constructed quadratic covariation processes may lie in the linear span of previously constructed ones and of the family, but may not lie in the linear span of repeated integrals of these. 
Remark 2: Property (B) is essential for the definition of the alphabet underlying the quasi-shuffle algebra in Section 4. In order that the multiplication of semimartingales defines a quasi-shuffle product, the nested covariation processes have to be assigned new letters in the alphabet except if they are in the linear span of other letters in the alphabet (that is if they are linear combinations of previously constructed quadratic covariation processes and the semimartingales themselves). Property (B) excludes families of semimartingales for which quadratic covariation processes would correspond to words, that is multiple integrals, rather than letters in the quasi-shuffle algebra.

Example 2.3 We give several examples to illustrate the concept of minimality.

(a) Suppose the family of semimartingales $\mathcal{X}$ is generated by independent Wiener processes $W^{1}, \ldots, W^{d}$. Then $\left[W^{i}, W^{j}\right]_{t}=\delta_{i j} t$ and $\left[W^{i}\right]^{(n)}=0$ for all $n \geq 3$. Thus $\mathcal{X}$ is minimal.

(b) Suppose the family of semimartingales $\mathcal{X}$ is generated by independent Poisson processes $P^{1}, \ldots, P^{d}$. Then $\left[P^{i}, P^{j}\right]=\delta_{i j} P^{i}$, and $\left[P^{i}\right]^{(n)}=P^{i}$ for all $n \geq 2$. Thus $\mathcal{X}$ is minimal.

(c) More generally, we will show in Section 3 that independent Lévy processes with moments of all orders generate a minimal family of semimartingales.

(d) Let $X_{t}^{1}=W_{t}$ and $X_{t}^{2}=\int_{0}^{t} W_{s} \mathrm{~d} s$, where $W$ is a Wiener process. Then $\left[X^{1}, X^{1}\right]_{t}=t$, and $X_{t}^{2}=\int_{0}^{t} X_{s}^{1} \mathrm{~d}\left[X^{1}, X^{1}\right]_{s}$. Hence $\left\{X^{1}, X^{2}\right\}$ is not minimal. However, the family generated by $\left\{X^{1}\right\}$ only is minimal.

\section{Minimal families of Lévy processes}

We consider the case of $d$ independent Lévy processes $X^{1}, \ldots, X^{d}$. We will assume here and in the sequel that all $X^{i}$ have moments of all orders. Without loss of generality, assume that all $X^{i}$ are stochastic. The goal of this section is to show that $\mathcal{X}=\left\{X^{1}, \ldots, X^{d}\right\}$ is a minimal family. If one of the processes $X^{i}$ is continuous, the process $t$ will be generated by $\left[X^{i}\right]^{(2)}$. Otherwise, if none of the $X^{i}$ is continuous, then we augment $\left\{X^{1}, \ldots, X^{d}\right\}$ with $t$, and $\left\{t, X^{1}, \ldots, X^{d}\right\}$ will be minimal.

Recall that a Lévy process is zero at time 0 , has independent stationary increments and is continuous in probability. Since the processes $X^{i}$ are independent, condition (A) in the definition of a minimal family is satisfied. To show that condition (B) is fulfilled, we assume first that $d=1$, and write $X=X^{1}$. The Lévy process $X$ can be characterised by the triplet $\left(\alpha, \sigma^{2}, \nu\right)$, where $\alpha$ and $\sigma$ are constants and where $\nu$ is a measure on $\mathbb{R}$ with $\nu(0)=0$ satisfying $\int_{\mathbb{R}} \inf \left(1, x^{2}\right) \nu(\mathrm{d} x)<\infty$. The Lévy Decomposition Theorem (see e.g. Protter 1992 Theorem I.42) states that the process $X$ has a unique decomposition

$$
X_{t}=\alpha t+\sigma W_{t}+J_{t}
$$

where $W$ is a Wiener process and $J$ is a purely discontinuous martingale. Note in particular that Lévy processes are special semimartingales. In terms of the Lévy measure $\nu$, we can express $J$ in the form

$$
J_{t}=\int_{0}^{t} \int_{\mathbb{R}} x(Q(\mathrm{~d} s, \mathrm{~d} x)-\mathrm{d} t \nu(\mathrm{d} x))
$$

where $Q(\mathrm{~d} t, \mathrm{~d} x)$ is a random Poisson measure with intensity measure $\mathrm{d} t \times \nu(\mathrm{d} x)$. Theorem 1.4.52 in Jacod \& Shiryaev (2002) and Theorem I.36 in Protter (1992) 
imply that the quadratic variation and power brackets of $X$ are given by

$$
[X]_{t}^{(n)}=\sigma^{2} 1_{\{n=2\}} t+\sum_{0 \leq s \leq t}\left(\Delta J_{s}\right)^{n}=\sigma^{2} 1_{\{n=2\}} t+\int_{0}^{t} \int_{\mathbb{R}} x^{n} Q(\mathrm{~d} s, \mathrm{~d} x)
$$

for $n \geq 2$. Note that the power bracket is again a Lévy process, and that for $n \geq 2$ $E\left[[J]_{t}^{(n)}\right]=t \int_{\mathbb{R}} x^{n} \nu(\mathrm{d} x)<\infty$, see Nualart \& Schoutens (2000, p. 111). For $n \geq 2$, set $\alpha_{n}=\int_{\mathbb{R}} x^{n} \nu(\mathrm{d} x)$, and denote the compensated power jump processes by

$$
Y_{t}^{(1)}=[X]_{t}^{(1)}-\alpha t \quad \text { and } \quad Y_{t}^{(n)}=[J]_{t}^{(n)}-\alpha_{n} t .
$$

The processes $Y^{(n)}, n \geq 1$, are known as the Teugels martingales, see Nualart \& Schoutens (2000). The power bracket has thus the unique decomposition $[X]_{t}^{(n)}=$ $\left(\sigma^{2} 1_{\{n=2\}}+\alpha_{n}\right) t+Y_{t}^{(n)}$ as the sum of the purely discontinuous martingale $Y^{(n)}$ and the deterministic process $\left(\sigma^{2} 1_{\{n=2\}}+\alpha_{n}\right) t$.

Two locally square-integrable martingales are strongly orthogonal, if their (real) product is a local martingale (see Jacod \& Shiryaev 2002, I.4.11 Definition). Since $X$ is assumed to have moments of all orders, all compensated power processes $Y^{(n)}$ are square-integrable martingales. Hence one can find pairwise strongly orthogonal square-integrable martingales $H^{(i)}, i \geq 1$, such that

$$
Y^{(n)}=c_{n, 1} H^{(1)}+c_{n, 2} H^{(2)}+\ldots+H^{(n)}
$$

for $n \geq 1$, where $c_{n, i}$ are constants with $c_{n, n}=1$, see Nualart \& Schoutens (2000).

Remark 1: A standard procedure to construct the strongly orthogonal martingales $H^{(n)}$ is as follows (see Davis \& Varaiya 1974, Davis 2005, Nualart \& Schoutens 2000 and Jamshidian 2005). For a locally square-integrable martingale $M$, there exists a unique predictable increasing process $\langle M, M\rangle$, the sharp or angular bracket of $M$, such that $M^{2}-\langle M, M\rangle$ is a local martingale. By direct calculation, the sharp bracket of the Teugels martingales $Y^{(i)}$ and $Y^{(j)}$ is given by $\left\langle Y^{(i)}, Y^{(j)}\right\rangle_{t}=\left(\alpha_{i+j}+\sigma^{2} 1_{\{i=j=1\}}\right) \cdot t$, see equation (1.7) in Davis (2005). Define inductively $H^{(1)}:=Y^{(1)}$ and $H^{(n)}:=Y^{(n)}-\sum_{k=1}^{n-1} \int \frac{\mathrm{d}\left\langle Y^{(n)}, H^{(k)}\right\rangle}{\mathrm{d}\left\langle H^{(k)}, H^{(k)}\right\rangle} \mathrm{d} H^{(k)}$ for $n \geq 2$. Importantly, the sharp brackets $\left\langle H^{(k)}, H^{(k)}\right\rangle_{t}$ are scalar multiples of $t$. It follows for a square-integrable process $\varphi$ we have $\left\|\int_{0}^{t} \varphi_{s} \mathrm{~d} H_{s}^{(n)}\right\|_{L^{2}(P)}=0$ for all $t \geq 0$ if and only if $\varphi \equiv 0$. A standard localisation procedure now implies the following identity, which is essential for the proof of Theorem 3.2. Let $n \geq 1$, and assume that $H^{(1)}, \ldots, H^{(n)} \not \equiv 0$. If $\varphi^{i}, i=1, \ldots, n$, are left-continuous processes with $\sum_{i=1}^{n} \int \varphi_{s}^{i} \mathrm{~d} H_{s}^{(i)} \equiv 0$, then $\varphi^{i} \equiv 0$ for all $i=1, \ldots, n$. We remark that in essence the equality $\sum_{i=1}^{n} \int \varphi_{s}^{i} \mathrm{~d} H_{s}^{(i)} \equiv 0$ describes the Galtchouk-Kunita-Watanabe decomposition of the martingal that is identically zero into the sum of $n$ orthogonal locally square-integrable stochastic integrals $\int \varphi_{s}^{i} \mathrm{~d} H_{s}^{(i)}$. This decomposition is known to be unique, see the remark on page 127 following Theorème 4.27 in Jacod (1979).

Lemma 3.1: Let $k \geq 1$. Consider the following properties:

(a) $H^{(k)} \equiv 0$.

(b) $Y^{(k)}$ is in the linear span of $\left\{Y^{(1)}, \ldots, Y^{(k-1)}\right\}$.

(c) $[X]^{(k)}$ is in the linear span of $\left\{t,[X]^{(1)}, \ldots,[X]^{(k-1)}\right\}$.

(d) $[X]^{(n)}$ is in the linear span of $\left\{t,[X]^{(1)}, \ldots,[X]^{(k-1)}\right\}$ for all $n \geq k$.

(e) $Y^{(n)}$ is in the linear span of $\left\{Y^{(1)}, \ldots, Y^{(k-1)}\right\}$ for all $n \geq k$. 
(f) $H^{(n)} \equiv 0$ for all $n \geq k$.

Then (a) implies properties (b) to (f). Furthermore (a), (b) and (c) are equivalent, and $(d),(e)$ and $(f)$ are equivalent.

Proof: The equivalence of (a), (b) and (c) and the equivalence of (d), (e) and (f) follow directly from the definitions and relations between the power bracket processes, the Teugels martingales and the orthogonal basis. We will show that (c) implies (d). Suppose that $[X]^{(k)}$ is in the linear span of $\left\{t,[X]^{(1)}, \ldots,[X]^{(k-1)}\right\}$. By definition we have $[X]^{(k+1)}=\left[X,[X]^{(k)}\right]$, and hence $[X]^{(k+1)}$ is in the linear span of $\left\{t,[X]^{(1)}, \ldots,[X]^{(k)}\right\}$, which by assumption on $[X]^{(k)}$ coincides with the linear span of $\left\{t,[X]^{(1)}, \ldots,[X]^{(k-1)}\right\}$. Hence, we conclude inductively that $[X]^{(n)}$ is in the linear span of $\left\{t,[X]^{(1)}, \ldots,[X]^{(k-1)}\right\}$ for all $n \geq k$.

Remark 2: The implication from (c) to (d) holds for general semimartingales.

Property (B) for the minimality of the family $\{X\}$ follows from the following stronger key property of the power brackets of $X$.

Theorem 3.2: Let $n \geq 1$, and assume there are left-continuous processes $\varphi^{k}$, $k=0, \ldots, n-1$, such that

$$
[X]^{(n)}=\sum_{k=1}^{n-1} \int \varphi_{s}^{k} \mathrm{~d}[X]_{s}^{(k)}+\int \varphi_{s}^{0} \mathrm{~d} s
$$

Then $\varphi^{k}$ is constant for all $k=0, \ldots, n-1$.

Proof: We can assume without loss of generality that $H^{(1)}, \ldots, H^{(n-1)} \neq 0$. If $H^{(k)} \equiv 0$ for some $k \geq 1$, then $[X]^{(j)}$ is in the linear span of $\left\{t,[X]^{(1)}, \ldots,[X]^{(k-1)}\right\}$ for all $j \geq k$ by Lemma [3.1, and equation (31) is equivalent to $[X]^{(n)}=$ $\sum_{k=1}^{k_{0}} \int \psi_{s}^{k} \mathrm{~d}[X]_{s}^{(k)}+\int \psi_{s}^{0} \mathrm{~d} s$, where $\psi^{k}$ are suitably defined left-continuous processes, and where $k_{0}=\min \left\{k \geq 1: H^{(k)} \equiv 0\right\}$. By compensating the power brackets, equation (3) is equivalent to

$Y_{t}^{(n)}+\alpha_{n} t=\sum_{k=1}^{n-1} \int_{0}^{t} \varphi_{s}^{k} \mathrm{~d} Y_{s}^{(k)}+\sum_{k=2}^{n-1} \int_{0}^{t} \varphi_{s}^{k}\left(\alpha_{k}+\sigma^{2} 1_{\{k=2\}}\right) \mathrm{d} s+\int_{0}^{t}\left(\varphi_{s}^{1} \alpha+\varphi_{s}^{0}\right) \mathrm{d} s$.

The uniqueness of the decomposition of the stochastic integral into predictable finite variation process and local martingale yields that for all $t \geq 0$

$$
\alpha_{n}=\varphi_{t}^{1} \alpha+\varphi_{t}^{0}+\sum_{k=2}^{n-1} \varphi_{t}^{k}\left(\alpha_{k}+\sigma^{2} 1_{\{k=2\}}\right)
$$

and

$$
Y_{t}^{(n)}=\sum_{k=1}^{n-1} \int_{0}^{t} \varphi_{s}^{k} \mathrm{~d} Y_{s}^{(k)}
$$

The latter equation is equivalent to $\sum_{i=1}^{n} c_{n i} H^{(i)}=\sum_{k=1}^{n-1} \sum_{i=1}^{k} \int \varphi_{s}^{k} c_{k i} \mathrm{~d} H_{s}^{(i)}$, with $c_{n n}=1$, and after rearrangement $H^{(n)}-\sum_{i=1}^{n-1} \int \sum_{k=i}^{n-1} \varphi_{s}^{k} c_{k i}-c_{n i} \mathrm{~d} H_{s}^{(i)}=0$. Since $H^{(i)} \not \equiv 0$, we have by Remark 1 that $\sum_{k=i}^{n-1} \varphi^{k} c_{k i}-c_{n i} \equiv 0$ for all $i=1, \ldots, n-1$. 
Recursively, it follows that $\varphi^{k}$ is constant for all $k=n-1, \ldots, 1$. Equation (4) now implies that $\varphi^{0}$ is constant. Hence the assertion follows.

Corollary 3.3: The family $\{X\}$ is minimal.

Remark 3: For $n \geq 2$ the power bracket $[X]^{(n)}$ has finite variation. Hence, any integral representation of $[X]^{(n)}$ cannot include an integral with respect to the Wiener process $W$. Hence if $\sigma \neq 0$, then the integrand $\varphi^{1}$ in (3) must be zero.

Suppose for the moment that $d=2$, and let $X$ and $Z$ be independent Lévy processes with Lévy decompositions $X_{t}=\alpha t+\sigma W_{t}+J_{t}$ and $Z_{t}=a t+c B_{t}+L_{t}$, where $B$ and $W$ are independent Wiener processes and $J$ and $L$ are independent purely discontinuous martingales. In particular, the power brackets $[X]^{(n)}$ and $[Z]^{(m)}$ are independent for all $n, m$. Hence, any representation of the power bracket $[X]^{(n)}$ as a sum of stochastic integrals cannot have non-zero contributions from the process $Z$ and its power brackets as long as these are stochastic, it can only have contributions through the deterministic process $t$, if any. Hence $\mathcal{X}=\{X, Z\}$ is a minimal family. This property extends straightforwardly to $d$ independent Lévy processes. In conclusion, we have established the following.

Theorem 3.4: $\quad$ Let $X^{1}, \ldots, X^{d}$ be independent non-deterministic Lévy processes with moments of all orders. Then $\left\{X^{1}, \ldots, X^{d}\right\}$ is a minimal family of semimartingales. If none of the $X^{i}, i=1, \ldots, d$, is continuous, then $\left\{t, X^{1}, \ldots, X^{d}\right\}$ is also a minimal family.

The following lemma completely characterises Lévy processes with power brackets that are linear combinations of lower order power brackets as compound Poisson processes which assume a finite number of values only (with or without continuous component $\left.\alpha t+\sigma W_{t}\right)$. As an important consequence we have that the alphabet underlying the quasi-shuffle algebra in Section 4 is finite if and only if the purely discontinuous martingale part of each $X^{i}, i=1, \ldots, d$, is a linear combination of (standard) compensated Poisson processes.

Proposition 3.5: Let $X_{t}=\alpha t+\sigma W_{t}+J_{t}$. Let $n \geq 1$. If the $n$-power bracket $[X]^{(n)}$ is in the linear span of $\left\{t,[X]^{(1)}, \ldots,[X]^{(n-1)}\right\}$, then the Lévy measure $\nu$ of $X$ has finite support of at most $n-1$ points. In other words, there exist an integer $k \leq n-1$ and constants $a_{1}, \ldots, a_{k}$ such that

$$
X_{t}=\alpha t+\sigma W_{t}+\sum_{i=1}^{P_{t}} Y_{i}-\alpha_{1} t
$$

where $P$ is a standard Poisson process, the $Y_{i}, i \in \mathbb{N}$, are independent identically distributed random variables, independent of $P$, with values in $\left\{a_{1}, \ldots, a_{k}\right\}$, and $\alpha_{1}:=\int_{\mathbb{R}} x \nu(\mathrm{d} x)$. Conversely, if $X$ has the form given in equation (6), then $[X]^{(n)}$ is in the linear span of $\left\{t,[X]^{(2)}, \ldots,[X]^{(k+1)}\right\}$ for all $n \geq k+2$.

Proof : First assume there are constants $c_{0}, c_{1}, \ldots, c_{n-1}$ such that $[X]_{t}^{(n)}=c_{0} t+$ $\sum_{k=1}^{n-1} c_{k}[X]^{(k)}$. It follows that $(\Delta X)^{n}=\sum_{k=1}^{n-1} c_{k}(\Delta X)^{k}$. Hence the jumps of $X$ satisfy $(\Delta X)^{n-1}-\sum_{k=1}^{n-1} c_{k}(\Delta X)^{k-1} \equiv 0$. There are at most $n-1$ distinct real roots to this equation, say $a_{1}, \ldots, a_{k}$ with $k \leq n-1$. Thus the support of the Lévy measure $\nu$ of $X$ is the set $\left\{a_{1}, \ldots, a_{k}\right\}$. The Lévy decomposition theorem (Protter, Theorem IV.42) implies the jump component $J$ of $X$ is a compensated compound Poisson process $J_{t}=\sum_{i=0}^{P_{t}} Y_{i}-\alpha_{1} t$, where $Y_{i}, i \in \mathbb{N}$, is a sequence of independent random variables with values in $\left\{a_{1}, \ldots, a_{k}\right\}$.

Second, for the converse result, assume that $X$ has the form stated. For $n \geq 2$ the $n$-power bracket is given by $[X]_{t}^{(n)}=\sum_{i=1}^{P_{t}} Y_{i}^{n}+\sigma^{2} 1_{\{n=2\}} t$. Let 
$c_{1}, \ldots, c_{k+1}$ be constants such that $a_{1}, \ldots, a_{k}$ are the real roots of the polynomial $c_{1}+c_{2} x+\ldots+c_{k+1} x^{k}$. Then $c_{k+1} Y_{i}^{k}=-c_{1}-c_{2} Y_{i}^{1}-\ldots-c_{k} Y_{i}^{k-1}$, and hence $c_{k+1} Y_{i}^{k+2}=-c_{1} Y_{i}^{2}-c_{2} Y_{i}^{3}-\ldots-c_{k} Y_{i}^{k+1}$. It follows that $c_{k+1}[X]_{t}^{(k+2)}=$ $c_{k+1} \sum_{i=1}^{P_{t}} Y_{i}^{k+2}=-\sum_{j=2}^{k+1} c_{j-1} \sum_{i=1}^{P_{t}} Y_{i}^{j}=-\sum_{j=2}^{k+1} c_{j-1}[X]_{t}^{(j)}+c_{1} \sigma^{2} t$. Hence $[X]^{(k+2)}$ and consequentially all higher power brackets $[X]^{(n)}$ for $n \geq k+2$ are linear combinations of $t,[X]^{(2)}, \ldots,[X]^{(k+1)}$.

Remark 4: Since compound Poisson processes that assume finitely many values can be expressed as linear combinations of independent standard Poisson processes, a Lévy process $X$ of the form in equation (6) is given equivalently by $X_{t}=\alpha t+\sigma W_{t}+a_{1} \bar{P}_{t}^{1}+\ldots+a_{k} \bar{P}^{k}(t)$, where $\bar{P}^{i}$ are independent compensated Poisson processes, $i=1, \ldots, k$.

\section{Construction of a quasi-shuffle algebra}

We recall the definition of a quasi-shuffle algebra following the exposition in Hoffman and Ihara (2012).

Let $\mathbb{A}$ be a countable alphabet. Let $\mathbb{R} \mathbb{A}$ denote the vector space with $\mathbb{A}$ as basis. We suppose there is commutative associative product $[\cdot, \cdot]$ on $\mathbb{R} \mathbb{A}$. Let $\mathbb{R}\langle\mathbb{A}\rangle$ denote the algebra of noncommutative polynomials and formal power series over the field of real numbers $\mathbb{R}$ generated by monomials (or words) $w=a_{1} a_{2} \cdots a_{n}$ with $a_{i} \in \mathbb{A}$.

Definition 4.1: (Quasi-shuffle product) The quasi-shuffle product $*$ is defined recursively on $\mathbb{R}\langle\mathbb{A}\rangle$ via $v a * w b=(v * w b) a+(v a * w) b+(v * w)[a, b]$, where $v$ and $w$ are words and $a$ and $b$ are letters.

Definition 4.2: For a minimal family of semimartingales $\mathcal{X}=\left\{X^{1}, \ldots, X^{d}\right\}$, we define the countable alphabet $\mathbb{A}$ inductively as follows:

(1) $\mathbb{A}$ contains the letters $1, \ldots, d$.

(2) Inductively, for $n \geq 2$ and for $k_{1} \leq k_{2} \leq \ldots \leq k_{n} \in\{1, \ldots, d\}$ consider the nested quadratic covariation process $\left[X^{k_{1}},\left[X^{k_{2}},\left[\ldots\left[X^{k_{n-1}}, X^{k_{n}}\right] \ldots\right]\right.\right.$. If this process is not in the linear span of $\mathcal{X}$ and previously constructed ones, then assign it a new letter.

Remark 1: In general the alphabet $\mathbb{A}$ is not finite.

Let $\mu: \mathbb{A} \rightarrow \mathcal{A}$ denote the map that identifies a letter with the corresponding semimartingale in $\mathcal{X}$ or one of the power bracket processes identified in (2) above. Let $\mathbb{R}\langle\mathbb{A}\rangle$ denote the set of all noncommutative polynomials and formal series on the alphabet $\mathbb{A}$ over $\mathbb{R}$. We extend the map $\mu$ defined above as follows: for a word $w=a_{1} \ldots a_{n} \in \mathbb{R}\langle\mathbb{A}\rangle$ with letters $a_{1}, \ldots, a_{n}$ we set $\mu(w)=I_{w}$ where $I_{w}$ is the multiple integral $I_{w}(t) \equiv \int_{0}^{t} \cdots \int_{0}^{\tau_{n-1}-} \mathrm{d} I_{a_{1}}\left(\tau_{n}\right) \cdots \mathrm{d} I_{a_{n}}\left(\tau_{1}\right)$ with $I_{a_{i}}=\mu\left(a_{i}\right), i=1, \ldots, n$, i.e. if $a \in\{1, \ldots, d\}$ then $I_{a}=X^{a}$ or if $a$ is from (2) then $I_{a}$ is a nested quadratic covariation process. We extend this to $\mathbb{R}\langle\mathbb{A}\rangle$ linearly. We can now pullback the multiplication of multiple integrals to define a product on $\mathbb{R}\langle\mathbb{A}\rangle$ as follows. For words $v$ and $w$ and letters $a$ and $b$, the product of multiple integrals satisfies $I_{v a} I_{w b}(t)=\int_{0}^{t} I_{v}(\tau-) \int_{0}^{\tau-} I_{w}(s-) \mathrm{d} I_{b}(s) \mathrm{d} I_{a}(\tau)+$ $\int_{0}^{t} \int_{0}^{\tau-} I_{v}(s-) \mathrm{d} I_{a}(s) \cdot I_{w}(\tau-) \mathrm{d} I_{b}(\tau)+\int_{0}^{t} I_{v}(\tau-) I_{w}(\tau-) \mathrm{d}\left[I_{a}, I_{b}\right](\tau)$. Recall that the bracket process defines a commutative, associative product on the real vector space generated by $\mathbb{A}$ (see Remark 1 ). Thus the pullback under $\mu$ defines a quasi-shuffle product $*$ on $\mathbb{R}\langle\mathbb{A}\rangle$ where we set $[a, b]:=\mu^{-1}([\mu(a), \mu(b)])$. We summarize our 
findings in the following key theorem.

Theorem 4.3: The map $\mu$ is an algebra isomorphism between the quasi-shuffle algebra $(\mathbb{R}\langle\mathbb{A}\rangle, *)$ and the algebra generated by the minimal family $\left\{X^{1}, \ldots, X^{d}\right\}$.

We now give $(\mathbb{R}\langle\mathbb{A}\rangle, *)$ an additional structure via a grading.

Definition 4.4: (Grading) On the alphabet $\mathbb{A}$ we define a grading $g$ as follows: For $a \in\{1, \ldots, d\}$, we only require $g(a) \in \mathbb{N}$, e.g. $g(a)=1$. For a letter $a \in \mathbb{A}$ with $\mu(a)=\left[X^{i}, X^{j}\right]$, set $g(a)=g(i)+g(j)$. More generally, for a letter $a \in \mathbb{A}$ that is mapped under $\mu$ to a nested quadratic covariation process, set $g(a)$ to be the sum of the gradings of each of its components.

Remark 2: For each $n \geq 1$ there are only finitely many letters of grade $n$. Thus $\mathbb{A}$ equipped with the grading $g$ is locally finite. We extend the grading $g$ to words $w=a_{1} \ldots a_{n}$ by setting $g(w)=g\left(a_{1}\right)+\ldots+g\left(a_{n}\right)$. Then $(\mathbb{R}\langle\mathbb{A}\rangle, *)$ equipped with the grading $g$ is a filtered quasi-shuffle algebra, i.e. the quasi-shuffle product of any two words $v$ and $w$ is a linear combination of words with degree $g(v)+g(w)$ or less (Lang 2002; p. 172). An algebra equipped with a grading $g$ is a graded algebra, if for any two words $v$ and $w$, the product $v * w$ is a linear combination of words with degree $g(v)+g(w)$ (Lang 2002; p. 172). Hence $(\mathbb{R}\langle\mathbb{A}\rangle, *)$ is a graded algebra if and only if for any two letters $a, b \in \mathbb{A}$ with $[a, b] \neq 0$ the grading $g$ satisfies $g([a, b])=g(a)+g(b)$.

Corollary 4.5: (Lévy Processes) Suppose that $\left\{X^{1}, \ldots, X^{d}\right\}$ are independent (non-deterministic) Lévy processes that have finite moments. Then:

(a) The algebra generated by the minimal family $\mathcal{X}=\left\{X^{1}, \ldots, X^{d}\right\}$ is isomorphic to the quasi-shuffle algebra $\mathbb{R}\langle\mathbb{A}\rangle$, where the alphabet $\mathbb{A}$ is defined via (1) and (2) above. If none of the $X^{i}$ is continuous, then this holds true for the minimal family $\mathcal{X}=\left\{X^{0}, X^{1}, \ldots, X^{d}\right\}$ with $X_{t}^{0}:=t$.

(b) The alphabet $\mathbb{A}$ in (a) is finite, if and only if for each $i=1, \ldots, d$, the purely discontinuous martingale part of $X^{(i)}$ is either identically zero or a linear combination of independent standard compensated Poisson processes.

(c) Suppose that the grading $g$ is specified on $\{0,1, \ldots, d\}$ as $g(0)=2$ and $g(i)=1$ for $i=1, \ldots, d$. Then the algebra $(\mathbb{R}\langle\mathbb{A}\rangle, *)$ equipped with the grading $g$ is a graded algebra unless the purely discontinuous martingale part of one or more of the $X^{(i)}, i=1, \ldots, d$, is a linear combination of independent standard compensated Poisson processes.

Proof: Result (a) follows from Theorem 3.4 and result (b) from Proposition 3.5, For result (c), we have to show that for any letters $a, b \in \mathbb{A}$ with $[a, b] \neq 0$ the grading $g$ satisfies $g([a, b])=g(a)+g(b)$. Since $\left[X^{i}, X^{j}\right] \equiv 0$ for $i \neq j$, we can assume that $a$ and $b$ have corresponding semimartingales $\mu(a)=\left[X^{i}\right]^{(n)}$ and $\mu(b)=$ $\left[X^{i}\right]^{(m)}$. We have by definition $\mu([a, b])=\left[\left[X^{i}\right]^{(n)},\left[X^{i}\right]^{(m)}\right]=\left[X^{i}\right]^{(n+m)}$. If $X^{i}$ is continuous, say $X_{t}^{i}=\alpha t+\sigma W_{t}$, then $\left[X^{i}\right]^{(n+m)}=\sigma^{2} 1_{\{n=m=1\}} \cdot t$. Hence if $\sigma \neq 0$ then $g([a, b])=2$ for $n=m=1$, and $[a, b]$ is zero otherwise. Hence result (c) follows for continuous $X^{i}$. Suppose now that $X^{i}$ is not continuous. By definition of $g$ we have $g([a, b])=(n+m) g(i)=g(a)+g(b)$, if $\left[X^{i}\right]^{(n+m)}$ is not in the linear span generated by $t$ and $\left[X^{i}\right]^{(k)}$ with $k \leq n+m-1$. On the other hand if $\left[X^{i}\right]^{(n+m)}$ is in the linear span generated by $t$ and $\left[X^{i}\right]^{(k)}$ with $k \leq n+m-1$, then $[a, b]$ is a linear combination of letters with degree of at most $(n+m-1) g(i)$. By Proposition [3.5] the power bracket $\left[X^{i}\right]^{(n+m)}$ is in the linear span generated by $t$ and lower order power brackets if and only if the purely discontinuous martingale part of one or more of the $X^{(i)}, i=1, \ldots, d$, is a linear combination of independent standard 
compensated Poisson processes. Hence assertion (c) follows.

\section{Conclusions and further work}

The main results we have proved, that minimal families of semimartingales form a quasi-shuffle algebra and a family of independent Lévy processes generate such a minimal family, are important in their own right. However, there are important further implications and applications we intend to pursue, see Curry et al. (2013). First the Hoffman exponential map gives an isomorphism between the shuffle and quasi-shuffle algebras. This simplifies the algebra and analysis and raises a natural question. Would the corresponding shuffle algebra be based on the Marcus integral (see Marcus 1981 or Applebaum 2009)? Second, with deconcatenation as a coproduct and a natural antipode established therefrom, the quasi-shuffle algebra becomes a Hopf algebra. Thus in principle we can establish the quasi-shuffle convolution algebra of endomorphisms on the quasi-shuffle Hopf algebra. See Reutenauer (1993, p. 58) for the shuffle case and Novelli, Patras and Thibon (2011) for the quasishuffle case. The convolution algebra is a natural setting for designing numerical methods for stochastic differential equations. See Ebrahimi-Fard et al. (2012) where efficient numerical methods for stochastic differential equations driven by Wiener processes are constructed utilizing the convolution shuffle algebra. Hence our next goal is to construct efficient numerical methods for stochastic differential equations driven by Lévy processes.

\section{References}

1. Applebaum, D. 2009 Lévy Processes and Stochastic Calculus. Cambridge Studies in Advanced Mathematics 116, Second Edition, CUP.

2. Curry, C., Ebrahimi-Fard, K., Malham, S.J.A., Wiese, A. 2013 Efficient integrators for systems driven by Lévy processes. In preparation.

3. Davis, M.H.A. 2005 Martingale Representation and All That. In: Abed, E.H. (Ed.). Advances in Control, Communication Networks, and Transportation Systems. Boston, etc: Birkhäuser, 57-68.

4. Davis, M.H.A., Varaiya, P. 1974 The multiplicity of an increasing family of $\sigma$-fields. The Annals of Probability 2(5), 958-963.

5. Duffaut Espinosa, L.A., Gray, W.S., González, O.R. 2012 On Fliess operators driven by $L_{2}$-Itô processes. Stochastics 84, 507-532.

6. Ebrahimi-Fard, K., Guo, L. 2006 Quasi-shuffles, Mixable Shuffles, and Hopf Algebras. Journal of Algebraic Combinatorics 24(1), 83-101.

7. Ebrahimi-Fard, K., Lundervold, A., Malham, S.J.A., Munthe-Kaas, H., Wiese, A. 2012 Algebraic structure of stochastic expansions and efficient simulation. Proc. R. Soc. A 468, 2361-2382.

8. Gaines, J. 1994 The algebra of iterated stochastic integrals, Stochastics and Stoch. Reports 49, 169-179

9. Hoffman, M.E. 2000 Quasi-shuffle products. Journal of Algebraic Combinatorics 11, 49-68.

10. Hoffman, M.E., Ihara, K. 2012 Quasi-shuffle products revisited. Preprint. Max-Planck-Institut für Mathematik Bonn.

11. Jacod, J. 1979 Calcul Stochastique et Problèmes de Martingales. Lecture Notes in Mathematics $\mathbf{7 1 4}$. Berlin, etc: Springer.

12. Jacod, J., Shiryaev, A.N. 2002 Limit Theorems for Stochastic Processes. Berlin, etc: Springer.

13. Jamshidian, F. 2005 Chaotic expansion of powers and martingale representation. Preprint.

14. Jamshidian, F. 2011 On the combinatorics of iterated stochastic integrals. Stochastics 83, 1-15.

15. Lang, S 2002 Algebra. 3rd Revised Edition, Berlin, etc: Springer.

16. Li, C.W., Liu, X.Q. 1997 Algebraic structure of multiple stochastic integrals with respect to Brownian motions and Poisson processes. Stochastics and Stoch. Reports 61, 107-120.

17. Malham, S.J.A., Wiese, A. 2009 Stochastic expansions and Hopf algebras. Proc. R. Soc. A 465, 3729-3749 doi:10.1098/rspa.2009.0203.

18. Marcus, S.I. 1981: Modeling and approximation of stochastic differential equations driven by semimartingales. Stochastics 4, 223-245.

19. Novelli, J.C., Patras, F., Thibon, J.-Y. 2011: Natural endomorphisms of quasi-shuffle Hopf algebras. arXiv:1101.0725 1. To appear in Bull. Soc. Math. de France.

20. Nualart, D., Schoutens, W. 2000 Chaotic and predictable representations for Lévy processes. Stochastic Processes and Their Applications 90, 109-122.

21. Protter, P. 1992 Stochastic Integration and Differential Equations. Berlin, etc: Springer.

22. Reutenauer, C. 1993 Free Lie algebras. London Mathematical Society Monographs New Series 7. Oxford Science Publications. 